\title{
FD 177:Mapping Atomic Motions with Ultrabright Electrons: Towards Fundamental Limits in Space-Time Resolution
}

Article in Faraday Discussions · November 2014

DOI: $10.1039 / C 4 F D 00204 K$

CITATIONS

11
READS

41

20 authors, including:

\section{Alexander Marx}

Max Planck Institute for the Structure and Dy...

53 PUBLICATIONS $\quad \mathbf{2 , 1 4 8}$ CITATIONS

SEE PROFILE

\section{Shima Bayesteh}

Deutsches Elektronen-Synchrotron

2 PUBLICATIONS 13 CITATIONS

SEE PROFILE
Lai Chung Liu

University of Toronto

12 PUBLICATIONS 107 CITATIONS

SEE PROFILE

\section{H. Delsim-Hashemi}

Deutsches Elektronen-Synchrotron

55 PUBLICATIONS $\quad 1,030$ CITATIONS

SEE PROFILE 


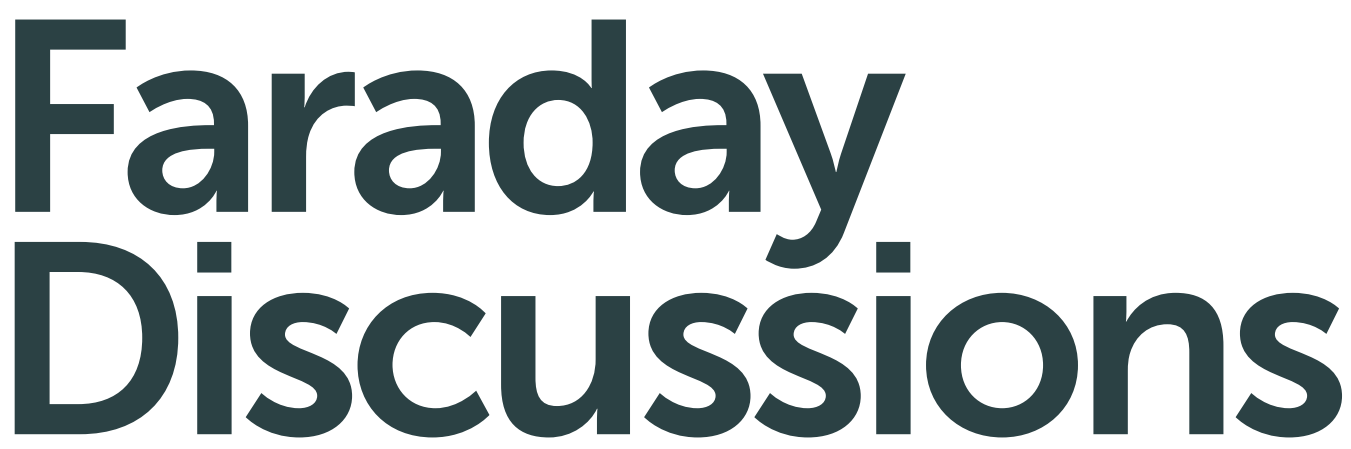

\section{Accepted Manuscript}

This manuscript will be presented and discussed at a forthcoming Faraday Discussion meeting. All delegates can contribute to the discussion which will be included in the final volume.

Register now to attend! Full details of all upcoming meetings: http://rsc.li/fd-upcoming-meetings

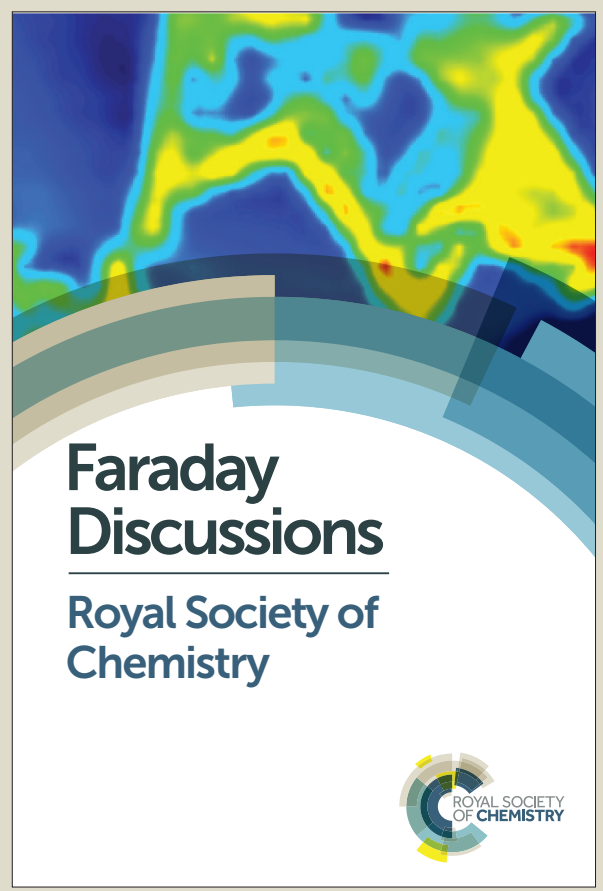

This is an Accepted Manuscript, which has been through the Royal Society of Chemistry peer review process and has been accepted for publication.

Accepted Manuscripts are published online shortly after acceptance, before technical editing, formatting and proof reading. Using this free service, authors can make their results available to the community, in citable form, before we publish the edited article. We will replace this Accepted Manuscript with the edited and formatted Advance Article as soon as it is available.

You can find more information about Accepted Manuscripts in the Information for Authors.

Please note that technical editing may introduce minor changes to the text and/or graphics, which may alter content. The journal's standard Terms \& Conditions and the Ethical guidelines still apply. In no event shall the Royal Society of Chemistry be held responsible for any errors or omissions in this Accepted Manuscript or any consequences arising from the use of any information it contains.

This article can be cited before page numbers have been issued, to do this please use: R. J. D. Miller, S. Manz, A. Casandruc, D. Zhang, Y. Zhong, R. Loch, A. Marx, T. Hasegawaa, L. C. Liu, S. Bayesteh, H. Delsim-Hashemi, M. Hoffmann, M. Felber, M. Hachmann, F. Mayet, J. Hirscht, S. Keskin, M. Hada, S. Epp and K. Flöttmann, Faraday Discuss., 2014, DOI: 10.1039/C4FD00204K. 


\author{
SCHOLARONE ${ }^{\text {m }}$ \\ Manuscripts
}




\title{
Mapping Atomic Motions with Ultrabright Electrons: Towards Fundamental Limits in Space-Time Resolution ${ }^{\dagger}$
}

\author{
Stephanie Manz, ${ }^{a}$ Albert Casandruc, ${ }^{a}$ Dongfang Zhang, ${ }^{a}$ Yin- \\ ${ }_{5}$ peng Zhong, ${ }^{a}$ Rolf A. Loch, ${ }^{a}$ Alexander Marx, ${ }^{a}$ \\ Taisuke Hasegawa, ${ }^{a, b}$ Lai C. Liu, ${ }^{c}$ Shima Bayesteh, ${ }^{d}$ Hossein \\ Delsim-Hashemi, ${ }^{e}$ Matthias Hoffmann, ${ }^{e}$ Matthias Felber, ${ }^{e}$ Max \\ Hachmann, ${ }^{e}$ Frank Mayet, ${ }^{e}$ Julian Hirscht, ${ }^{a}$ Sercan Keskin, ${ }^{a}$ \\ Masaki Hada, ${ }^{a, f}$ Sascha W. Epp, ${ }^{a}$ Klaus Flöttmann, ${ }^{e}$ and R.J. \\ Dwayne Miller ${ }^{a, c, g, *}$
}

\author{
Received Xth $X X X X X X X X X X 20 X X$, Accepted Xth XXXXXXXXX 20XX \\ First published on the web Xth $X X X X X X X X X X 200 X$ \\ DOI: $10.1039 / \mathbf{c 0 0 0 0 0 0 x}$
}

The long held objective of directly observing atomic motions during the defining 15 moments of chemistry has been achieved based on ultrabright electron sources that have given rise to a new field of atomically resolved structural dynamics. This class of experiments requires not only simultaneously sub-atomic spatial resolution with temporal resolution on the 100 femtosecond time scale but also has brightness requirements approaching single shot atomic resolution conditions. The brightness condition is in recognition that chemistry leads generally to irreversible changes in structure during the experimental conditions and that the nanoscale thin samples needed for electron structural probes pose upper limits to the available sample or "film" for atomic movies. Even in the case of reversible systems, the degree of excitation and thermal effects require the brightest sources 25 possible for a given space-time resolution to observe the structural changes above background. Further progress in the field, particularly to the study of biological systems and solution reaction chemistry, requires increased brightness and spatial coherence, as well as an ability to tune the electron scattering cross-section to meet sample constraints. The electron bunch density or intensity depends di-

30 rectly on the magnitude of the extraction field for photoemitted electron sources and electron energy distribution in the transverse and longitudinal planes of electron propagation. This work examines the fundamental limits to optimizing these parameters based on relativistic electron sources using re-bunching cavity concepts that are now capable of achieving 10 femtosecond time scale resolution to

35 capture the fastest nuclear motions. This analysis is given for both diffraction and real space imaging of structural dynamics in which there are several orders of magnitude higher space-time resolution with diffraction methods. The first experimental results from the Relativistic Electron Gun for Atomic Exploration (REGAE) are given that show the significantly reduced multiple electron scat-

40 tering problem in this regime, which opens up micron scale systems, notably solution phase chemistry, to atomically resolved structural dynamics. 


\section{Introduction}

One of the long standing objectives in science has been to have sufficient spatial and temporal resolution to directly watch atomic motions during the primary motions governing structural transitions ${ }^{1,2}$. This quest is relevant to helping under-

5 stand at the atomic level of detail, effectively, all classes of structural transitions from issues of condensed matter physics of strongly correlated electron-lattice materials, extreme states of matter, to biological functions. In chemistry, this objective is central to the discipline. The very notion of a chemical process involves the passage of a system from one stable structure to another in which the

10 chemist tries to control conditions to direct the desired process over the myriad of other possible outcomes. The ability to control chemistry largely resides in arranging conditions to lower the barrier and entropic factors to favour a particular chemical pathway. The intellectual pursuit of chemistry is to understand the factors that control barrier heights, within the context of a complex many body

15 potential, and to connect molecular structure to macroscopic properties. This statement is intended to include advances in ab initio theory, synthetic strategies, and experimental methods to probe different aspects of the system and systembath interactions driving chemistry. The unifying concept in this pursuit is to try to identify the key atomic motions that lead the system over the barrier re-

20 gion, or stated differently to picture the transition state region, the critical "point of no return" that connects the reactant to the product free energy surfaces. A direct observation of atomic motions during the transition from one structure to another would provide the most rigorous test possible for various concepts that have evolved for inferring chemical pathways. We could see this directly. This 25 objective has now been met $^{3-5}$. The recent development of ultrabright electron and X-ray sources to provide the necessary structural probes have opened up the femtosecond time domain to atomically resolved dynamics ${ }^{2-8}$. The source technology is rapidly advancing to enable atomic imaging of structural dynamics of ever larger and more complex systems. There are, however, fundamental limits

30 to source brightness and sample issues that need to be overcome to apply this new imaging modality to problems of general interest.

For the purpose of this Discussion, we will focus on source requirements with respect to spatial and temporal resolving power to study molecular systems undergoing chemical reactions. With respect to time resolution, the relevant time

35 scales to this problem have been well appreciated since the first connection of

${ }^{a}$ The Max Planck Institute for the Structure and Dynamics of Matter, Center for Free Electron Laser Science, Luruper Chaussee 149, Hamburg 22761, Germany.

${ }^{b}$ Department of Chemical Engineering, Faculty of Engineering Kyoto University Katsura, Nishikyoku, Kyoto, 615-8510, Japan.

${ }^{c}$ Departments of Chemistry and Physics, University of Toronto, Toronto, Ontario M5S 3H6, Canada.

${ }^{d}$ Institut für Experimentalphysik, Universität Hamburg, Luruper Chaussee 149, Hamburg 22761, Germany.

${ }^{e}$ DESY, Notkestrasse 85, 22607 Hamburg, Germany.

${ }^{f}$ Materials \& Structures Laboratory, Tokyo Institute of Technology, Yokohama 226-8503, Japan \& JST-PRESTO, Kawaguchi 332-0012, Japan.

$g$ The Hamburg Centre for Ultrafast Imaging CUI, Universität Hamburg, Luruper Chaussee 149, Hamburg 22761, Germany.

2 | Faraday Discuss., [year], [vol], 1-25

This journal is @ The Royal Society of Chemistry [year] 
microscopic processes to reaction rates in the quest for an absolute rate theory ${ }^{9}$. This work ultimately led to the development of transition state theory. The defining moment of chemistry is captured in the discussion of barrier crossing processes. This timescale varies for different systems but is on the order of few $10 \mathrm{~s}$ 5 of femtoseconds (fs; $1 \mathrm{fs}=10^{15} \mathrm{~s}$ ) to 100 femtosecond time scales. There are faster motions in nature. For example, the $\mathrm{OH}$ stretch of water has a period of 10 fs but the motions involved are less than $0.05 \AA$, hardly chemically relevant. The time scale is defined by the relevant motions over length scales corresponding to 0.1 to $1 \AA$, leading to distinct changes in structure. More specifically, these 10 motions must in turn be coupled to the nuclear continuum of states to relax the system to a displaced minimum in the many body potential corresponding to a long lived state or stable product state. There must be damping or energy dissipation in the process to relax on the product surface. It is the approximately $100 \mathrm{fs}$ time scale of relaxation along a reaction coordinate that dictates the required time 15 resolution. It is important to realize that not all motions are equally coupled to the reaction coordinate and the degree of coupling rapidly evolves in the barrier crossing region ${ }^{4}$. The highly anharmonic nature of the many body interactions at this far from equilibrium point in nuclear configuration space leads to strong mixing of the otherwise suitable normal mode basis to describe equilibrium fluc-

20 tuations. Normal modes are accurate descriptions of atomic motions for small excursions over which the potential is well approximated to be harmonic, i.e. the nuclear fluctuations are within linear response limits. The far from equilibrium fluctuations that sample the barrier crossing region are highly nonlinear and the most anharmonic modes tend to be the low frequency modes that undergo the 25 largest relative motions. These modes are also the most highly damped as they occur within the highest spectral density of modes for conserving energy and momentum in the relaxation process ${ }^{10,11}$. It is the net excursion along the modes most strongly coupled to the reaction coordinate that define the relevant time scales of chemistry. These modes, whether involving intramolecular processes, 30 solvent controlled chemistry, or the chemistry controlled by protein environmental fluctuations, tend to be in the $100 \mathrm{~cm}^{-1}$ frequency range, which gives rise to the generalized requirement of $100 \mathrm{fs}$ time resolution to capture the chemistry ${ }^{4}$.

The spatial resolution requirements are equally challenging in that one needs sub-Å spatial resolution to pull out the important relative motions directing atomic 35 displacements from one stable structure to another. To a first approximation, the spatial resolution requirement exceeds that needed for static structure determination. For example, in the photo-isomerization of retinal, the primary event involved in vision and energy transduction within the rhodopsin family of proteins, the key motions are on the order of $0.1 \AA$ for the carbon atoms at the central bond 40 axis of the isomerization ${ }^{12}$. Save in the act of bond dissociation, chemically relevant motions are between $0.1-1 \AA$. To fully resolve the primary motions requires either hard X-rays or high energy electron probes with sufficiently short carrier wavelengths to resolve these motions. Even so, this level of spatial resolution would be out of range for most sources, however, time-resolved measurements 45 involve differential measurements. The initial structure is known. It is only the relative changes from this known starting point that need to be resolved, not the entire structure for every atomic position to this level of accuracy. The key to being able to resolve chemically relevant motions is that the source serving as 
the structural probe must be sufficiently stable and bright enough to render signal to noise ratios (SNR) sufficient to pull out these small relative changes in intensity that report the atomic positions. The most sensitive method for determining atomic structure is the use of diffraction methods or imaging in reciprocal 5 space. This observable takes advantage of the $\mathrm{N}^{2}$ amplification of the diffracted signal intensity by scattering off $\mathrm{N}$ identically arranged molecules to amplify the scattered signal. The criteria of high brightness and high stability for the source reduce to achieving sufficient signal to noise ratios in the diffracted orders to pull out the structural changes of interest. The quality of sample often plays a 10 deciding factor in the achievable resolution; however, it is only recently that the sources serving as structural probes have reached the level of brightness where sample issues are the main bottleneck.

Differential detection of the structural changes is achieved by comparing the changes in structure following an excitation pulse, which triggers the structural 15 change of interest. Here, it needs to be fully appreciated that, in the absence of a "trigger", the act of barrier crossing driven by background thermal noise is a rare event. For even small barriers (e.g. $1 \mathrm{eV}$ ), there are less than 1:10 ${ }^{8}$ molecules undergoing a barrier crossing event at any instant in time ${ }^{4}$. In principle, one could track the motions of an individual molecule undergoing first order reac20 tions, or molecular collision partners for second order processes, to observe such crossings but the ability to observe such motions and the enormity of collecting sufficient atomic images at the required framing rate to get above background noise, never mind increased demands on source brightness, make this prospect intractable. The processes of interest must be optically triggered to observe the

25 key relative atomic motions connecting two stable structures within the complex, highly dimensional, potential energy surfaces of interest to chemistry. This simple realization has a number of important consequences for the conduct of the experiment. First, the perturbation used to trigger the chemistry must be faster than the ensuing motions of interest. Only femtosecond laser excitation meets 30 this requirement. Furthermore the optical excitation must prepare the system on excited state surfaces that intersect reactive crossings under barrierless conditions. If there is a barrier in the excited state, the time scale for the buildup of the product state is much slower than that of the primary motions involved. In the presence of a barrier, the system will reach a thermal equilibrium within the 35 vibrational modes corresponding to the excited state surface. In this event, there are uncorrelated, thermally, sampled crossings and the details of the relevant motions will not be resolved. The problem reverts to the same statistics as trying to capture barrier crossing events along the ground state. This requirement for a barrierless excited state process not only limits the number of potentially tractable 40 systems for study but has additional consequences. To observe the relevant motions above the background of unexcited molecules requires that on the order of $10 \%$ of the molecules or lattice sites are undergoing the photo induced structural change. The quantum yield for the desired photoprocess must be at least this large and there is an upper limit to the degree of excitation. The $<100$ femtosec-

45 ond requirements for the excitation pulse necessarily leads to high peak powers, however, the peak power must be maintained at excitation levels on the order of $100 \mathrm{GW} / \mathrm{cm}^{2}$ or lower to avoid multiphoton ionization artifacts ${ }^{13}$. Basically, above this peak power multiphoton processes begin to dominate and even the de-

4 | Faraday Discuss., [year], [vol], 1-25

This journal is @ The Royal Society of Chemistry [year] 
gree of state preparation becomes ill defined as multiphoton excitation to higher lying excited states begin to also significantly contribute. For typical molecular densities, this latter condition means that samples must be on the order of a few microns or thinner or else the required excitation levels ( $>10 \%$ photoproduct 5 formation) lead to excessively high incident peak powers. These are nontrivial sample constraints as one must have sufficient surface area within this thickness restriction to attain adequate signal to noise to stitch together a movie of the atomic motions involved in the process of interest.

Of all the above discussed requirements, the most limiting is the requirement for approximately $10 \%$ of the system undergoing the phototriggered structural transition. Apart from gas phase systems, which can be rapidly exchanged between laser shots, this level of excitation leads to sample damage in a single shot. This statement is especially true for single crystals that give the highest structural resolution. Normally in diffraction experiments, the upper limit for source

15 brightness is determined by X-ray or electron induced damage. In this class of experiments, the main limitation with respect to sample damage is not the structural probe but the excitation process to trigger the chemistry that damages the sample. It is not enough to have simultaneous atomic resolution with $100 \mathrm{fs}$ time resolution or less but one must attain this experimental parameter space within 20 single shot conditions. Herein lies the real challenge. The source brightness for the structural probe must not only be capable of high space-time resolution but of sufficient intensity to achieve atomic resolution at or approaching single shot conditions ${ }^{4,14}$.

Enormous gains in source brightness have been achieved for both electron and X-ray sources to meet this condition. The major advance in X-ray source brightness was achieved through technical advances made in undulator technology that enabled scaling the Free-Electron Laser concept to the X-ray range. These sources are referred to as 4th Generation Light Sources or X-Ray Free Electron Lasers (XFELs). In comparison to 3rd Generation synchrotron sources,

30 XFELs represent an increase in source brightness of over almost ten orders of magnitude ${ }^{7}$. XFELs are not true laser resonators but are based on self amplification of spontaneous emission or SASE sources. As with SASE sources in the visible range, there are huge stochastic fluctuations in intensity and spectrum that make shot to shot normalizing essential to improve the SNR. In addition, there are 35 time jitter issues, with respect to synchronizing the laser excitation used to trigger structural changes and the RF phase of the electron acceleration, that gives rise to $200 \mathrm{fs}$ timing jitter. In principle, XFELs are capable of $<50 \mathrm{fs}$ time resolution using time stamping methods as another normalization step to extract the time dependent changes in diffraction efficiency ${ }^{15}$. To date, time-resolved structural 40 dynamics on the prerequisite time scales ( $100 \mathrm{fs}$ to picosecond) have not been realized with sufficient numbers of diffraction orders to follow structural changes. There has been a recent report of resolved structural changes, albeit not the actual transient structures, on the microsecond time scale ${ }^{16}$ and studies of a single diffraction order have been used to infer the role of the lattice in directing ma-

45 terial properties for strongly correlated electron lattice systems ${ }^{17}$. There remain a number of technical challenges in the normalization procedures required to attain sufficient SNR and also in the large number of crystal projections needed for X-ray structure determination, prior to the onset of X-ray induced damage, 
that have hindered efforts in this direction. Further, these sources are not dedicated facilities to this line of inquiry. The most important application for XFELs appears to be in the use of the high spatial coherence and brightness to enable nano- to micro-protein crystallography, prior to the onset of X-ray induced dam5 age $^{8}$. Recent developments in self-assembly of up to M-pixel crystal arrays or photochips ${ }^{18,19}$ (solid target solution to sample delivery) and aerosol injectors to give random orientations ${ }^{20}$ may solve the last technical obstacle in providing a general solution for collecting sufficient reciprocal space to stitch together atomic movies on the femtosecond timescale.

With respect to electron sources, there are inherent electron-electron repulsion or space charge effects that limit source brightness. This problem was readily appreciated and it was felt that it would not be possible to achieve the needed brightness with electron sources. In this regard, there have been proposals to achieve the necessary space-time resolution with low intensity sources, with sin-

15 gle electron pulses being the ultimate limit to completely avoid space charge limits in time resolution ${ }^{21,22}$. The basic problem is that one needs approximately $10^{5}-10^{6}$ electrons to have sufficient SNR to invert diffraction patterns to structures and approximately 2-3 orders of magnitude more for real space imaging. In the single electron limit, this requirement translates to over $10^{8}$ photoexcita20 tion events in the data collection process. It is not possible to have sufficient sample area for such a large number of excitation events, save for gas phase samples where other issues have limited the time resolution. One needs fully reversible systems that can withstand over $10^{6}$ photocyles between reactive excited and ground electronic states. The prospect of using single electrons remains an 25 elusive prospect with respect to achieving simultaneous femtosecond time resolution and sub- $\AA$ spatial resolution to structural transitions. To date, there has only been one report on time resolved dynamics in the single electron limit in which simple thermally excited acoustic phonons were followed on the $100 \mathrm{ps}$ timescale. This process is fully reversibly by its very nature as it does not involve 30 a structural change. This work rather reinforces the importance of brightness. In all cases, the brighter the source the better the space-time resolution is, as long as the time resolution is sufficient to follow the dynamics of interest. As will be seen below, ultrabright electron pulses on the order of $10 \mathrm{fs}$ are now possible, which provides sufficient time resolution to follow even the fastest nuclear mo-

35 tions. The first atomic movie with sufficient space-time resolution, i.e., sufficient number of diffraction orders, to resolve the relative atomic motions involved in a structural change was in fact captured with a high brightness electron source ${ }^{3}$. The major advance that made this possible was the realization that high bunch charge electron pulses do not lose space-time correlation at sufficient intensities 40 to achieve single shot structure determination. This realization was made possible through an effectively exact solution to the electron propagation dynamics by solving the coupled equations of motions of some $10^{4}$ electrons ${ }^{23}$, sufficient for the structure determination of systems with simple unit cells $(<3 \mathrm{~nm})$. It was discovered that the transverse velocity spread, related to the transverse spatial 45 coherence, was not significantly affected. The main issue was the longitudinal space charge effect. Two solutions were apparent from these calculations. One solution involved the use of extremely short propagation paths to the sample target to limit pulse broadening to retain 100 fs time resolution. Here, the key

6| Faraday Discuss., [year], [vol], 1-25

This journal is @ The Royal Society of Chemistry [year] 
realization was that the transverse coherence, even at short propagation distances with typical transverse velocity spreads, was sufficient for atomic resolution for systems with unit cells of a few $\mathrm{nm}$. The other solution to emerge from this work was to explicitly exploit the conserved space-time correlation and development 5 of an extremely linear chirp that naturally develops for nonrelativistic electrons to compress the pulse at the sample position. In this respect, the electrons at the front of the electron pulse experience electron-electron repulsion effects that lead to acceleration or energy exchange between the electrons at the back of the pulse that experience deceleration. For nonrelativistic electrons, the higher 10 energy electrons travel faster than the slow energy electrons. The electrons at the front stay at the front and the electrons at the back stay at the back to conserve the original space-time relationship and develop an extremely linear chirp with propagation. This observation led to the proposal for the use of electric field compression methods or other dispersive elements to compress the elec15 tron pulse ${ }^{23}$. The use of a longitudinal half cycle RF cavity to recompress the pulse conserves the transverse coherence and appears to be the best solution to pulse compression for high brightness applications ${ }^{24}$, although there are other applications for improved time resolution ${ }^{25}$. The overall gains in electron source brightness over previous low electron density pulsed sources approaches simi-

20 lar gains as XFELs over prior technology. The compact electron gun design is now capable of $10^{5}-10^{6}$ electrons per pulse in a $200 \mathrm{fs}$ pulse that is focusable down to 100 micron radius spot sizes typically used in femtosecond laser experiments $^{2}$. New designs at higher energies will enable $<100$ fs pulses. The DC-RF pulse compression concept has achieved approximately a factor of 10 increase in 25 brightness with attendant technical issues in RF timing jitter that currently limit the achieved time resolution to approximately $200 \mathrm{fs}^{2,26-28}$. The time resolution can be improved to $30 \mathrm{fs}$ with the use of time stamping methods ${ }^{29}$. In comparison, the compact electron gun is jitter free. These table top electron sources are exceptionally bright. For calibration, taking into account the factor of $10^{5}-10^{6}$

30 higher scattering cross-section for electrons over X-rays for the same energy, this source technology is comparable to $10^{12} \mathrm{X}$-ray photons per pulse for practical laser excitation parameters, i.e. these electron sources are on par with XFELs in terms of observed signal levels. Here it is important to keep in mind that peak power limitations require the use of samples on the micron scale or smaller such

35 that there is little distinguishing differences in sample requirements. The diffraction efficiency for X-rays is very small for sample thicknesses on the micron scale such that the observed signal levels are expected to be similar for these two different source technologies. The big differences are that the electron sources are dedicated table top facilities for this class of experiment and are orders of mag-

40 nitude more stable than the X-ray counterpart. It is these decided advantages that has enabled atomic movies of the primary motions involved in structural transitions to be first captured using electron sources. Advances in laser generated $\mathrm{X}$-ray plasma sources have likewise opened up atomically resolved structural dynamics on the prerequisite sub-ps timescale ${ }^{30-32}$. The most important difference

45 in brightness levels of XFELs sources in relation to all other sources is the near perfect transverse coherence. This difference makes XFELs the ideal source for the study of complex large unit cell systems such as proteins; whereas electron sources are currently limited to transverse coherences suitable for the study of 
relatively small proteins $(<10 \mathrm{~nm}$ unit cells). This difference may not hold for long, as will be discussed in this report. Electron sources have at least 2 orders of magnitude possible increase in brightness that may even close this gap in imaging resolution. In this respect, the recent introduction of relativistic electron sources 5 for this application ${ }^{33-38}$ promises to provide the highest spatial-temporal resolution due to the reduction of pulse broadening effects in the relativistic regime. The full potential of relativistic electron sources to achieve the highest possible time resolution relies on removing the initial velocity and temporal spread in the $\mathrm{RF}$ acceleration phase. This Discussion paper focuses on the first results from 10 the Relativistic Electron Gun for Atomic Exploration (REGAE) that introduces the use of a rebuncher cavity to achieve the ultimate limit in high brightness for relativistic electron sources.

\section{Ultrafast Diffraction and Real Space Imaging with Rela- tivistic Electrons}

\subsection{Coherence Issues: Diffraction}

The focus of this Discussion paper is on electron source technology for atomic imaging dynamics on the primary timescales of chemistry. The image resolution as with any source is related to both the transverse and spatial coherence of the source for diffractive imaging or aberrations in the lens system for real

20 space reconstruction. For X-rays or other light sources, the degree of coherence is defined by the beam divergence, which is a constant after the modality of light generation is fixed. For electrons, the degrees of transverse and longitudinal coherence are coupled parameters that depend on the specific space-time focusing. As an electron pulse is made shorter in duration for a given bunch charge at some

25 point the space charge effects and spatial inhomogeneity will lead to increased beam divergence or transverse velocity spread and corresponding loss of in-plane resolution. The transverse spatial coherence is defined by ${ }^{39-41}$ :

$$
L_{x} \approx \lambda / 2 \pi \sigma_{\theta} \approx \hbar / \sigma_{p_{x}},
$$

where $\sigma_{p_{z}}$ is the angular spread, and $\sigma_{p_{x}}$ is given by the transverse momentum spread. There is a similar relation for the longitudinal coherence, which is defined 30 as:

$$
L_{z} \approx \hbar / \sigma_{p_{z}} .
$$

The longitudinal coherence is only an issue if one wants to coherently reconstruct an image as in holography. For diffractive imaging, the longitudinal coherence is not the limiting factor. The coherence length, even for strongly space charge broadened pulses, is generally much larger than the unit cells of even large unit 35 cell crystals $(>10 \mathrm{~nm})$. Thus, the two most important, coupled, parameters to consider in attaining the required space-time resolution for a particular problem of interest is to match the transverse coherence to the unit cell of interest for the spatial resolution and to adjust the electron bunch density accordingly to give the required electron pulse duration for the time resolution. In the former case, 40 the required spatial resolution was achieved by adjusting the source size at the photocathode or introducing an aperture to produce an effective source size that 
gives a transverse coherence length at the sample position that is a few times the lateral dimension of the unit cell ${ }^{41}$. In terms of time resolution, the required spatial resolution limits the bunch density for a given transverse energy spread and the minimum pulse duration on target is achieved by reducing the number of 5 electrons for a given probe beam size at the sample position, until the longitudinal space charge effects are negligible relative to the time resolution needed. Again, these parameters are coupled.

For most problems of chemical interest, the pulse duration with sufficient electrons for single shot structure determination is the primary concern. The 10 shortest electron pulses are achieved with the highest extraction fields as this minimizes the pulse propagation time to the sample position and thereby minimizes space charge broadening of the time resolution. The highest extraction fields are achieved with RF acceleration methods that avoid charge accumulation and breakdown. Extraction fields an order of magnitude larger than DC electron 15 guns are possible. In addition, as one goes to higher electron energies, there is a reduction in space charge broadening due to the relativistic correction to the electron velocities. To first order, in the fully relativistic regime, all the electrons would travel at essentially the same velocity, near the speed of light. In this limit, the time broadening problem would reduce solely to issues related to the transverse coherence or transverse velocity spread that would increase with temporal compression in the longitudinal direction. What are the fundamental limits to space-time resolution with electrons? There are new developments both in the generation of ultrabright electrons sources for the shortest possible electron pulse durations/temporal resolution and photocathode concepts that promise to offer 25 orders of magnitude increases in transverse coherence. These higher energy electron sources also have greater penetration depths to put sample constraints completely on par with X-ray sources. We are entering into a new regime for electron sources. The order of magnitude higher field gradients possible with relativistic electron guns and enormous reduction of longitudinal space charge broadening 30 in the relativistic regime hold promise to provide the brightest sources possible for the highest possible spatial-temporal resolution. We highlight the promise to go beyond present limits in diffraction or reciprocal space imaging of chemical and biological problems - for which the dynamics are essential to understanding mechanism and functionality.

\subsection{Coherence Issues: Real Space Imaging - Dynamic High Energy Elec- tron Microscopy}

The incoherent nature of the spatial phase of the electron pulses across the beam profile presents more difficulties for real space imaging than for diffraction, that are hard to compensate. To acquire an image with reasonable signal to noise ratio 40 at the detector position, one aims for $10^{6}$ to $10^{7}$ electrons per acquisition, which needs to be matched to a typical sample area of a few $\mu \mathrm{m}^{2}$. The electron beam needs to be tightly focused to achieve this density. A tight focus on the other hand means a reduction of the local coherence length, which calls for an improved total coherence of the electron pulse from its source on. The photoemitted electron 45 image at the cathode has to be as close to point-like as possible. Laser spot sizes below $10 \mu \mathrm{m}$ are difficult to achieve at the cathode position inside a RF cavity. 


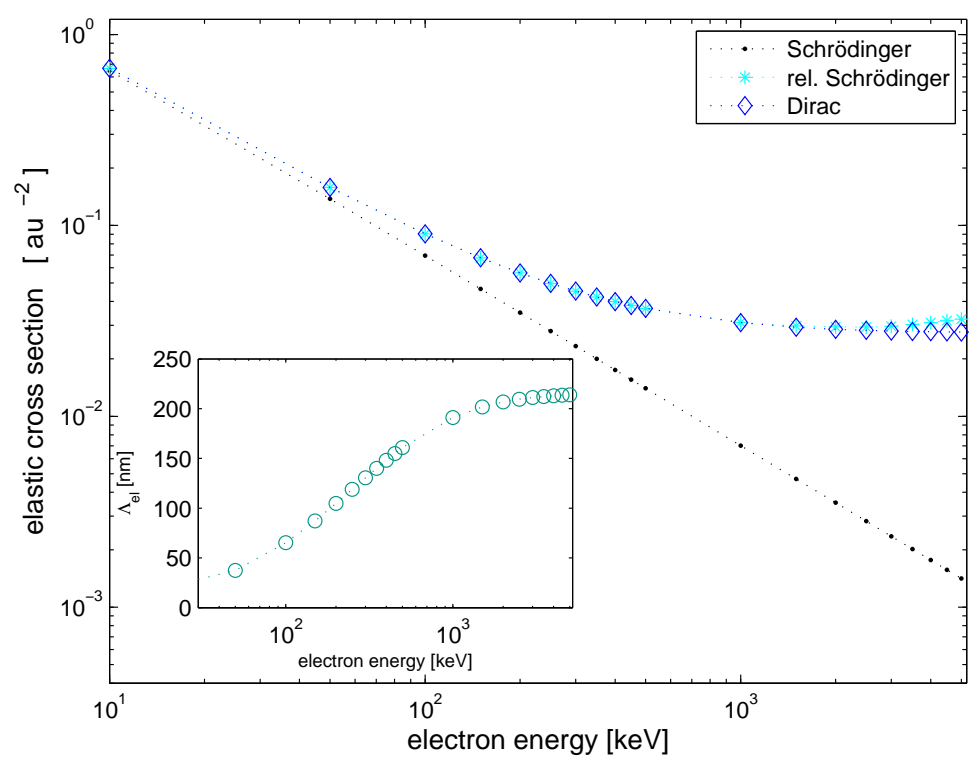

Fig. 1 Comparison of different methods to calculate electron cross-sections in Aluminum using the relations given in ref. ${ }^{42}$. The inset shows the according mean free path in Aluminum for the cross-sections derived from the Dirac equation.

Typically, the laser is coupled into the cavity from a window several tens of $\mathrm{cm}$ away from the cathode surface, which is insufficient working distance to focus tighter than 10 microns. It is possible to use structured cathodes to give the desired source size. However, for current designs, single shot imaging will only be 5 possible with a partially coherent mode and thus relies on $\mathrm{Z}$-contrast $\mathrm{Z}=$ atomic number) rather than phase contrast. By spatial filtering with an aperture, the scattered electrons lead to an intensity drop in the imaged electron beam relative to the position of the scattered object for bright-field imaging. In dark field imaging, the unscattered electron beam is stopped by a negative aperture (beam stop) and 10 the scattered electrons are imaged. In both cases, the favorable situation is for each electron to scatter less than once on average. Too little scattering gives low imaging contrast and calls for high electron doses, whereas multiple scattering broadens the minimal spot size of point scatterers in the sample. The number of scattering events depends on the elastic cross-section $\sigma_{e l}$, which depends on

15 the electrons' energy and the atomic number $\mathrm{Z}$ of the scattering material. An approximate expression for $\sigma_{e l}$ is given by ${ }^{43}$ :

$$
\sigma_{e l}=\frac{c^{2} h^{2} Z^{4 / 3}}{\pi E_{0} \beta}
$$

where $E_{0}$ is the electron rest energy, and $\beta=v / c$ with the electron's velocity $v$. The mean free path $\Lambda_{e l}$ of an electron in a material with density $\rho$ is related to the cross-section by $\Lambda_{e l}=A / N_{a} \sigma_{e l} \rho$. While the expression for $\sigma_{e l}$ gives a helpful 20 approximation, it can differ from the actual cross-section by a factor of 2 . We use the approach presented in ref. ${ }^{42}$ to include relativistic corrections in the calculation. In fig. 1, we compare calculations for the elastic cross-section using the 
Schrödinger equation, the relativistically corrected Schrödinger equation, as well as the Dirac equation. Solvers for both the relativistic Schrödinger equatation and Dirac equation have been implemented into the EDICo-code ${ }^{44}$. The calculations were performed for unpolarized electrons. We find no significant difference 5 between the relativistic Schrödinger and Dirac result and conclude that effects of spin can be neglected, as also stated in ref. ${ }^{42}$. In terms of beam propagation through the sample and effect on imaging, the electron beam broadens its energy distribution in the sample by inelastic collisions. Typically, the total crosssections for elastic and inelastic scattering show a qualitatively similar behavior with energy. The ratio $\eta=\sigma_{\text {inel }} / \sigma_{e l}$ was experimentally found to be $\eta=20 / Z^{45}$ and theoretically $\eta=26 / Z^{43}$. Since the cross-section for elastic and inelastic scattering follow similar behavior with energy, $\eta$ is almost constant in the $\mathrm{keV}$ to $\mathrm{MeV}$ range. One can assume that multiple scattering will be reduced by the same factor for both elastic and inelastic scattering. Damage due to ionization 15 will also be reduced by the same argument, but knock-on damage will be more severe than for lower energy devices.

When the sample thickness exceeds the mean free path in the material, one faces two problems in transmission electron microscopy that stem from multiple scattering: multiple elastic scattering increases the minimal spot size of a point 20 like object; and inelastic scattering similarly leads to aberrations in the reconstructed real space image. Thus, the resolution will be reduced and distorted. Such effects are reduced in electron microscopy with relativistic electrons, and allow in the case of light, low Z, organic materials, the study of micron thick samples with nanometer resolution. High resolution microscopy with thin samples

25 in which atomic resolution is desired may better be performed in conventional electron microscopes employing aberration correctors ${ }^{46}$ that are not currently available for relativistic electron imaging systems.

We studied the feasibility of dynamic real space imaging with pulse propagation simulations in ASTRA. The simulation is launched from the sample position so on. A pulse of $10^{4}$ particles with a Gaussian density profile and an energy of 3 $\mathrm{MeV}$ is tracked through the magnetic field of a realistic electromagnetic solenoid, which is a likely implementation for the objective lens. The solenoid field was calculated using the CST software suite ${ }^{47}$. A schematic of the imaging system implemented in the existing REGAE setup is shown in fig. 2(a). With the pulse,

35500 test particles were used to probe the mean field space charge that travel along the propagation direction. For a chosen spot size of $10 \mu \mathrm{m}$ at the sample, the test particles travel off-center at a distance of $1 \mu \mathrm{m}$. The energy spread for the pulse is chosen to be $2 \times 10^{-4}$. The transverse momentum spread of the test particles was chosen to match scattered electrons up to typical angles of $5 \mathrm{mrad}$. We per-

40 formed the simulation for three different focal lengths of $1.1 \mathrm{~cm}, 5 \mathrm{~cm}$, and 10 $\mathrm{cm}$. The results depending on the bunch charge are presented in fig. 2 (b). We tracked the test particles until they reached the image plane, and then computed the RMS width to obtain a point spread function. We find higher resolution for lower focal lengths, which is to be expected from the respective spherical aberra-

45 tion coefficients. Furthermore, we observe a decrease in resolution depending on charge, which we interpret as the result of the inhomogeneous space charge field in the bunch, an effect resembling spherical aberrations ${ }^{48}$. The simulation has been repeated for different pulse lengths and spot sizes. We find a similar scaling 


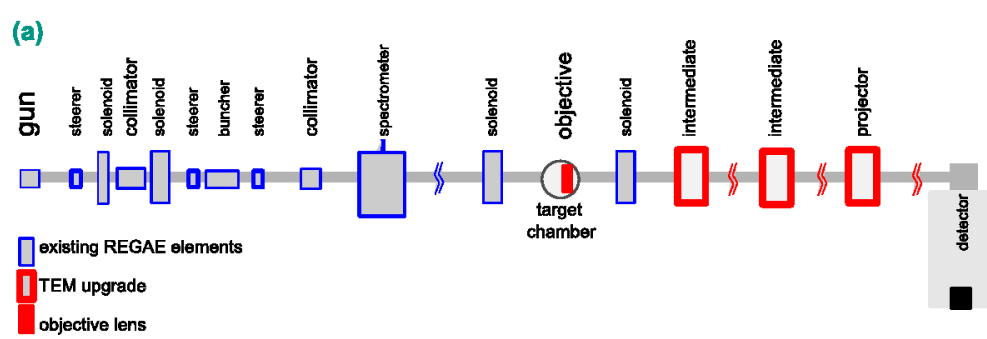

(b)

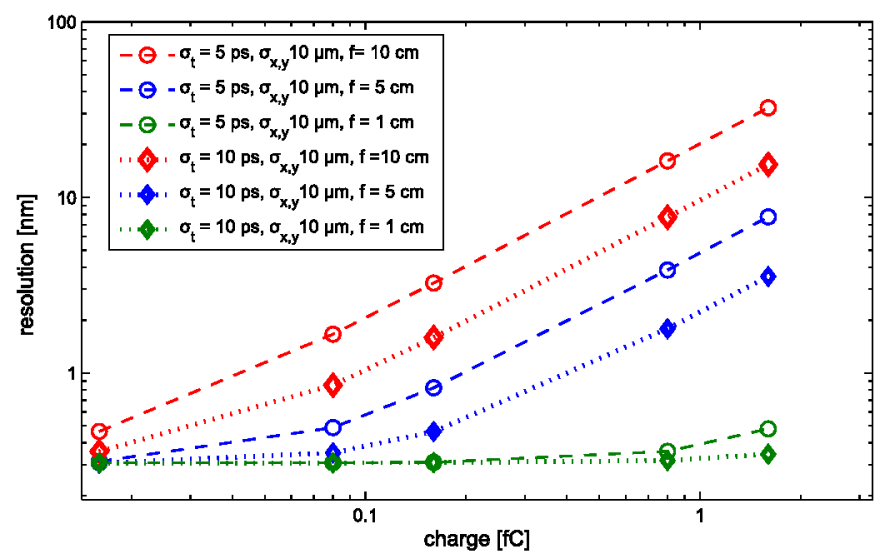

Fig. 2 (a) Schematic of the implementation of a TEM mode within the existing REGAE setup.(b) Effect of charge and objective focal length on resolution for a pulse length of 5 and 10 ps. The beam shape is Gaussian for all cases.

for all pulse shapes, as visible in fig. 2(b). In order to reach $1 \mathrm{~nm}$ resolution the charge must be kept in the fC range. Longer pulses would certainly relax the issue of space charge induced aberration, as proposed in ${ }^{49,50}$, but then additional, higher harmonic cavities need to be employed to decrease the energy spread that 5 comes with the generation of a long pulse in an RF cavity, which has not been treated in these works.

\subsection{Electron diffraction with REGAE}

\subsection{The REGAE apparatus}

The relativistic electron gun for atomic exploration, REGAE, is a radio frequency

(RF) accelerator based gun design operating in a pulsed mode with 50 bursts per second. It is mainly dedicated to the study of structural dynamics in solid, liquid and gas phase of organic and inorganic samples. As elucidated in the introduction, such a device has to fulfill the requirements of short pulses, high brightness, and high coherence at the same time. Electron pulses are created via photo-

15 emission by $266 \mathrm{~nm}, 500 \mathrm{fs}$ pulses of a frequency-tripled Ti:Sa laser impinging the cathode within a 1.5 cell S-band cavity. For the studies presented here, we use either molybdenum, gold, or CsTe as the cathode material. By means of a coaxial on-axis coupler, a travelling $3 \mathrm{GHz}$ S-band wave of some micro-second length launched by a klystron is fed in resonance into the gun cavity, where field

12 | Faraday Discuss., [year], [vol], 1-25

This journal is @ The Royal Society of Chemistry [year] 


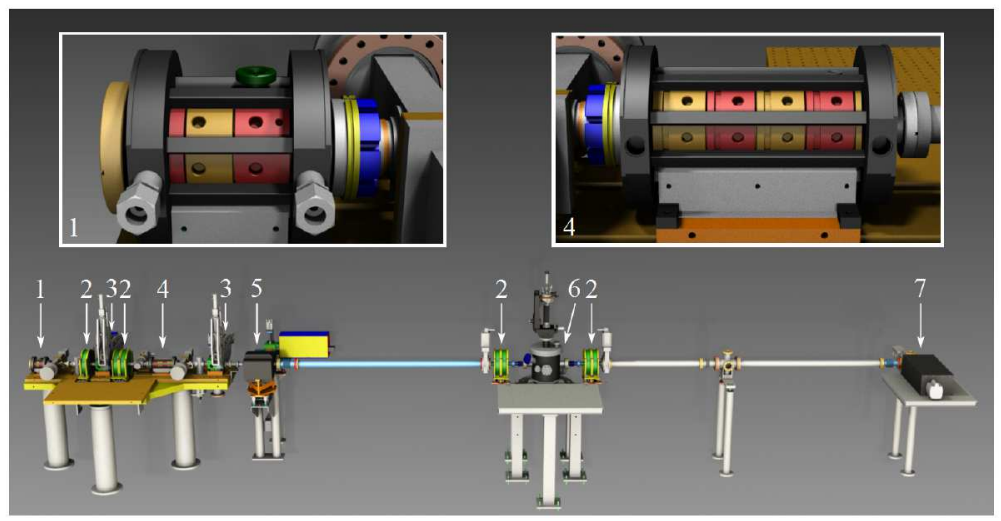

Fig. 3 REGAE scheme. Indicated with numbers are: 1) gun cavity, 2) solenoids, 3) collimators, 4) rebunching cavity, 5) dipole for electron energy measurements, 6) target interaction chamber, and 7) detector.

gradients of up to $100 \mathrm{MV} / \mathrm{m}$ can be obtained leading to electron energies up to $5 \mathrm{MeV}$. Running the cavity at high fields has, apart from the beam energy itself, the major advantage that high bunch charge densities can be extracted from the cathode overcoming conventional space charge limitations. Depending on the 5 cathode material and its quantum efficiency, bunch charges in the range from $\mathrm{pC}$ for gold and other metal cathodes and $10 \mathrm{pC}$ for CsTe can be extracted. For typical diffraction experiments, we aim for a bunch charges of 100-200 fC (about 1 million electrons) at the target position, as a compromise between beam quality and signal strength. The elements of the beamline are depicted in fig. 3 . The gun

10 section is followed by electro-magnetic solenoids and collimators to shape the beam. The collimators are a critical feature and require a high degree of engineering to ensure proper alignment under the ultrahigh vacuum conditions needed to run the RF cavity. These elements are used for collimation and beam clean up as well as to reduce dark current traveling in the beam direction. One of the most 15 important distinctions of this relativistic electron gun is the low dark current ${ }^{51}$ that is primarily due to the very low base pressure of $10^{-10}$ mbar employed in this design concept. Additional beam diagnostic stations allow for determination of transverse electron bunch parameters, e.g. beam width, energy, and energy spread. The beam charge can be detected without disturbing the beam by means 20 of a resonator, where the electron pulse induces a TM01 mode at $1.3 \mathrm{GHz}$. The bunch charge can be measured with fC resolution ${ }^{52}$. The other key feature to this design is the introduction of a rebunching cavity to obtain the shortest possible pulses at the sample position. The design employs the same klystron amplifier with an RF splitter to drive both cavities, which requires finding unique phase solutions for which the pulse is optimally compressed for a given amplification. The rebunching cavity is designed to temporally refocus the electron pulse at a distance of $5.5 \mathrm{~m}$ from the photocathode position. For detection, a scintillator on a fiber optics plate in the beam line converts the electrons' density distribution to photons, which are coupled to an EMCCD chip outside of vacuum. The high so dynamic range of the EMCCD combined with its high sensitivity down to single electron detection allows for simultaneous detection of the undiffracted beam and 
(a)

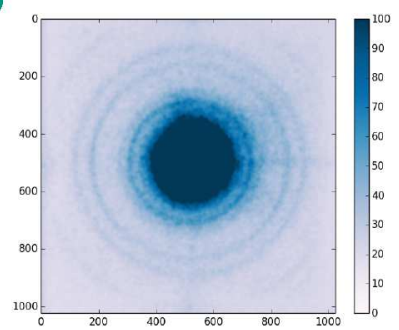

(c)

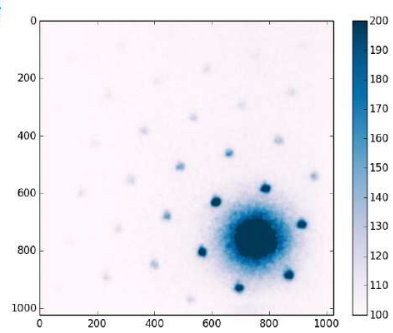

(b)

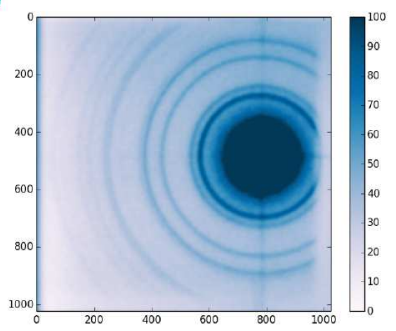

(d)

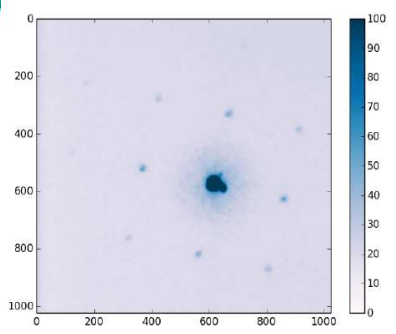

Fig. 4 Example diffraction patterns from (a,b): polycrystalline Gold, (c) : $\mathrm{MoS}_{2}$, (d) $\mathrm{Si}$ The quality of the diffraction is particularly notable for the single crystal samples $\mathrm{MoS}_{2}$ and Si.

diffraction patterns without the necessity for a beam block.

\subsection{Beam dynamics and time resolution at REGAE}

The beam dynamics after the gun are controlled by solenoid magnets, indicated by arrows in fig. 3. Depending on the demands on beam size at the sample posi-

5 tion and extent of the diffraction pattern on the detector screen, we either focus the beam through the target on the screen with solenoid lenses (see fig.3), or - if a higher intensity at the target is called for - have a small spot at the sample and refocus on the detector position. Ideal beam dynamics for diffraction in terms of a phase advance ${ }^{53}$ approaching $90^{\circ}$ has been found only without an intermediate 10 focus. Beam size, divergence, and coherence are controlled by the photocathode and gun cavity, and transverse beam shaping elements, as solenoids, collimators and steerers. At a distance of $1.3 \mathrm{~m}$ from the accelerator, a rebunching cavity is placed in the beam line. It consists of four cells and is operated off-crest compared to the arrival time of the electron bunch. The rebunching cavity is driven

15 by a fraction of the RF field that also supplies the gun. The relative phase between the cavities is adjusted with phase shifters. While the parameter space of this coupled cavity system is finite, efforts have to be made to overcome complications due to reflections and unwanted feedback between the two coupled cavities ${ }^{54}$. The electron bunch can, if higher acceleration is required, also be in-

${ }_{20}$ jected on-crest into the rebunching cavity. Potential beam parameters along the propagation axis are summarized in fig. 5. For this example, the beam dynamics for a bunch of $100 \mathrm{fC}$ is tracked by the ASTRA ${ }^{55}$ simulation software, which includes space charge fields. For the initial beam parameters, we use an RMS spot

14 | Faraday Discuss., [year], [vol], 1-25

This journal is @ The Royal Society of Chemistry [year] 

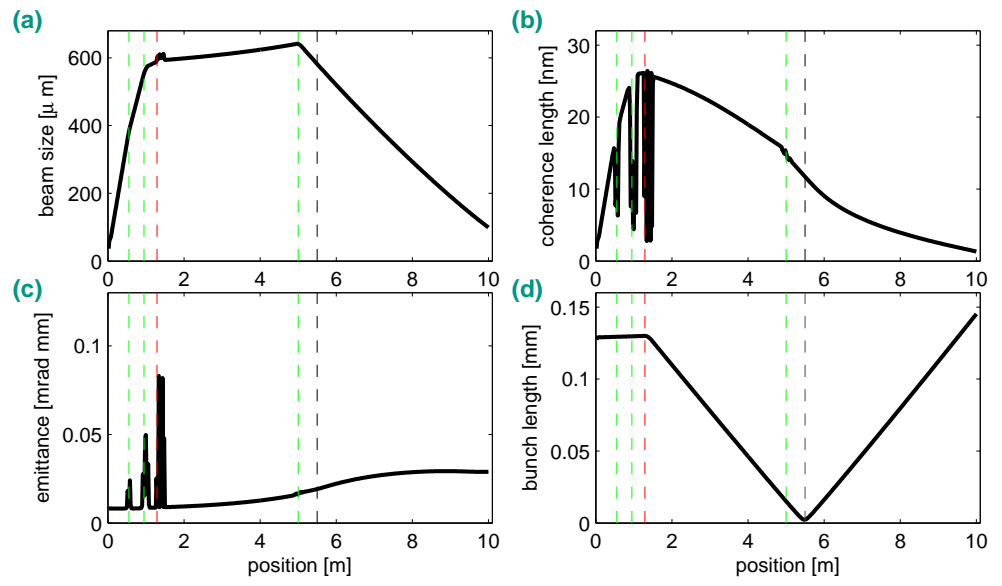

Fig. 5 ASTRA simulations for diffraction with REGAE: (a) RMS beam size showing the beam dynamics, (b) transverse coherence length, (c) emittance, and (d) bunch length in $\mathrm{mm}$, corresponding to $10 \mathrm{fs}$ at the longitudinal focus. The dashed lines indicate the position of solenoids (green), the rebunching cavity (red) and the sample position (black).

size of the laser at the cathode of $10 \mu \mathrm{m}$, a pulse length of $0.5 \mathrm{ps}$ and a charge of $100 \mathrm{fC}$. For this charge, the beam size at the target reaches $500 \mu \mathrm{m}$ with a transverse coherence length above $10 \mathrm{~nm}$. With the buncher tuned to the appropriate phase, a bunch length of $10 \mathrm{fs}$ can be expected at the longitudinal focus at the 5 sample position. We then use a solenoid close to the sample position to focus the beam and diffraction pattern to the detector. It needs to be emphasized that a pulse duration of $10 \mathrm{fs}$ is sufficiently short to capture even the fastest possible nuclear motions involved in chemical processes. The achievable transverse coherence is also notable in that the design objectives were to provide sufficient 10 transverse coherence to enable the study of systems as large as proteins. The unit cell of protein systems capable of being phototriggered to execute their biological functions such as heme proteins and the family of rhodopsin photoactive systems are on the order of $6 \mathrm{~nm}$ such that the transverse coherence is sufficient for this purpose.

\subsection{Static diffraction from Aluminum: thickness dependence}

One of the main limitations in the use of electron structural probes is the effect of multiple scattering due to the intrinsically high scattering cross-section of electrons. The increased electron penetration depth and reduced scattering angles for relativistic electrons promise the feasibility of ultra-fast electron diffraction (UED) with thick samples. In conventional electron microscopes and electron guns at intermediate energies (DC guns) typical sample thicknesses range from few tens of nanometers to $200 \mathrm{~nm}$, depending on the density and atomic weight of the material. However, many interesting systems, such as protein crystals, water soluble crystalline systems, and solution phase chemistry call for a probe with

25 a higher penetration depth. From the point of view of material science, a higher penetration depth supports the study of bulk properties with minimal effects from 

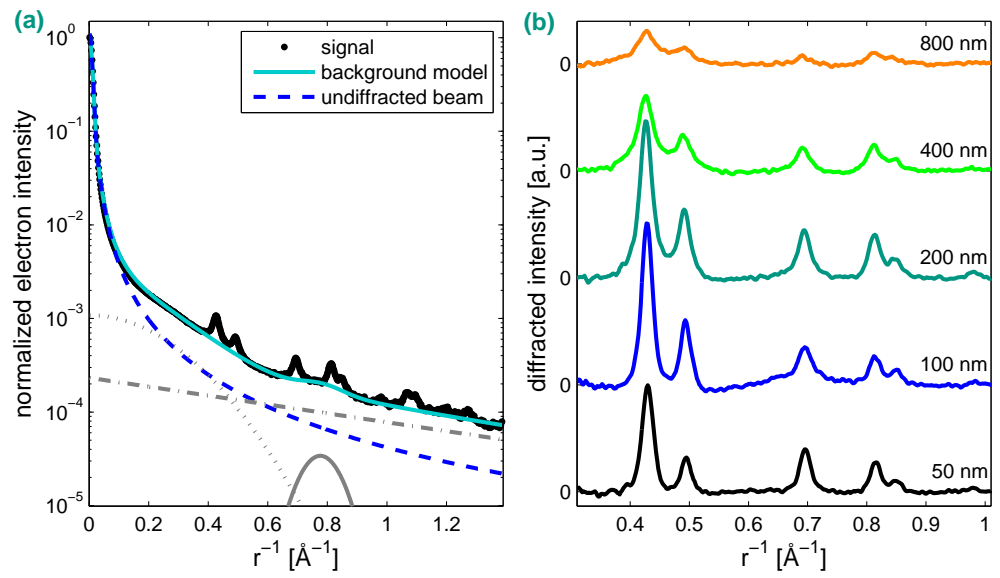

Fig. 6 (a) Example for electron diffraction from poly-crystalline Aluminum film after azimuthal integration. The signal (black) has been normalized to the transmitted peak intensity. A reference image including dark current has been subtracted before integration. Further subtraction removes the un-diffracted beam (dashed blue line) and incoherent background (solid blue line) from the coherently diffracted signal. Incoherent contributions are shown in grey. (b) Diffracted intensity after background subtraction. The diffraction patterns are stacked for better visibility.

interfaces or surface effects. The relativistic electron energy regime has not been extensively studied and, as discussed above, only approximate relationships in terms of scaling relations for elastic and inelastic scattering are available. The effect of multiple electron scattering on image resolution needs to be explicitly 5 determined. We explored these possibilities using films of polycrystalline Aluminum of increasing thickness up to $800 \mathrm{~nm}$. The calculated mean free path according to the inset in fig. 1 is just above $200 \mathrm{~nm}$. The films are supported by a $30 \mathrm{~nm}$ thin $\mathrm{SiN}$ membrane. Diffraction patterns were recorded by integrating 300 shots of $180 \mathrm{fC}$ pulses at $4 \mathrm{MeV}$. Before azimuthal integration of the diffrac-

10 tion pattern a background image is subtracted. The incoherent contribution and the diffuse diffraction ring from the SiN membrane are modelled with a multicomponent function and removed as well. These components are shown as gray lines in fig. 6(a). The off-center peak at about $0.78 \AA^{-1}$ corresponds to diffuse diffraction from the supporting SiN membrane. We need to determine the effect of multiple electron scattering as a function of thickness for a given system and use the $\mathrm{Z}$ dependence of the scattering to provide general guidelines for limiting this effect on reciprocal (diffraction) or real space resolution. Coherently scattered electrons interfere after multiple elastic scattering, thus, the diffracted intensities are altered compared to the single scattering regime. Inelastic scattering 20 leads to a broadening of both probe beam and diffracted electrons and can affect the resolution. The addition of both effects is visible as an increase of diffraction ring width, as shown in fig. 7(c). The question whether multi-scattering effects are necessary to describe such diffraction patterns in theoretical treatments or the degree to which structural information is lost are still open. Modulations in diffraction intensity might be averaged out in polycrystalline samples, as ex- 


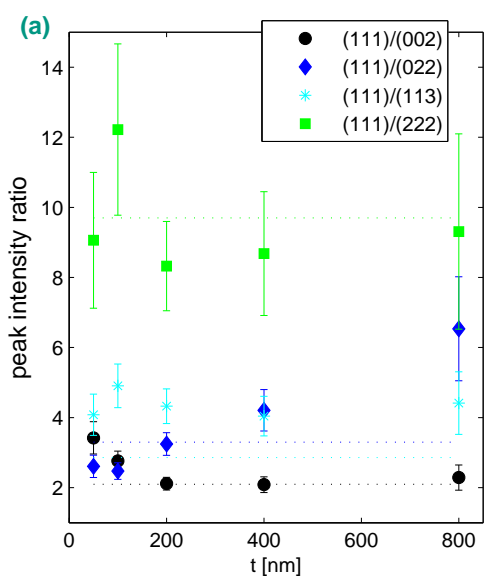

(b)

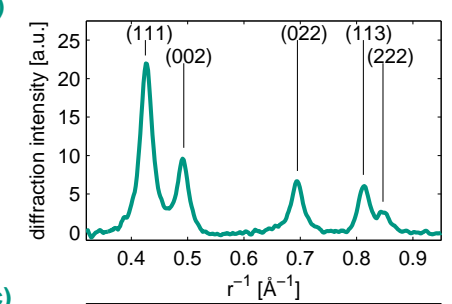

(c)

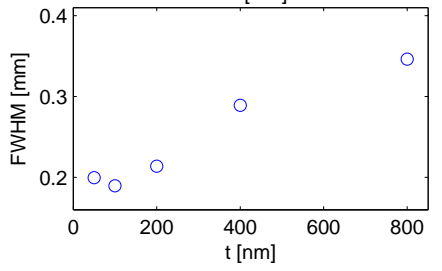

Fig. 7 (a) Ratio of peak intensities in diffraction pattern. The dashed lines gives the scattering ratio for a perfect powder sample in the single scattering regime for comparison. An example diffraction pattern for $200 \mathrm{~nm}$ thickness is shown in (b), where the integrated diffraction rings are labeled with Miller indices. (c) Width of rings in diffraction pattern with increasing thickness.

pected from multi-slice calculations. To aid in resolving this issue, we compare the ratio of diffraction intensities for different rings in fig. 7(a) with the expected result for a completely random powder sample, obtained from the software crystal maker ${ }^{56}$.The rather surprising result is that the multiple electron scattering 5 effects, while occurring, are not significantly altering the relative ratios of the different diffraction orders as needed for dynamic structure determination. The small deviation from ideal results is within signal to noise limits for some of the diffraction orders. To be fully conclusive, the effect of substructure, grain size and texture within the polycrystalline sample would need to be studied by a different method.

This study gives a direct determination of the effect of multiple electron scattering on a well-defined system. In the present case, $\mathrm{Al}$ is close in $\mathrm{Z}$ to organic systems. We conclude that time-resolved electron diffraction studies of systems, at least for light materials, is possible up to micrometer thickness in the rela15 tivistic energy range. Here is especially important to note that these experiments always deal with systems of known structure. The experimental challenge is to be able to discern small changes in the relative intensities of the different diffraction orders. This control study shows that it is possible to extract structural dynamics from samples as thick as several hundred nanometers to micron thickness at this 20 energy range. This feature of relativistic electrons was one of the primary motivations for developing this source as it dramatically increases the ease of sample preparation and opens up solution phase chemistry to atomic inspection, which is much easier to realize with micron scale pathlengths as opposed to $100 \mathrm{~nm}$ needed for nonrelativistic electron studies. 
(a)

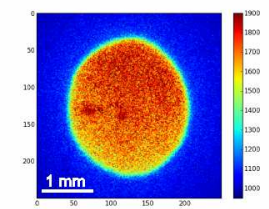

(b)

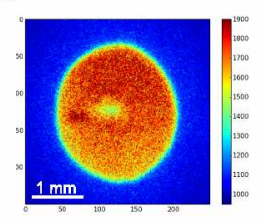

(c)

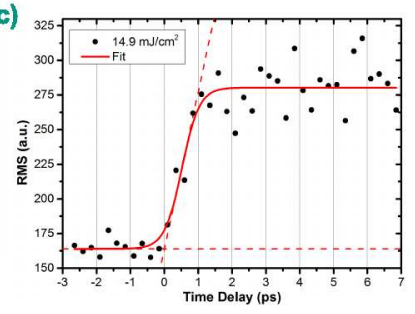

Fig. 8 Shadow images of a copper mesh before (a) and after (b) laser irradiation. The deflection of the electron bunch can be observed as a depleted region in the electron beam profile. The analysis of the signal is shown in (c): The signal RMS in the central region is evaluated and shows a step with a time constant of $0.9 \mathrm{ps}$.

\section{Ultrafast dynamics with REGAE}

\subsection{Finding $\mathbf{t}=\mathbf{0}$}

In order to perform time-resolved electron diffraction studies, one needs to characterize the instrument's time resolution. Before such studies can be performed

5 in detail however, the temporal overlap $(\mathrm{t}=0)$ between pump and probe, in our case excitation laser and electron pulse, needs to be found. Here, a process that is reversible under photo excitation is favorable. We used the laser induced plasma formation as a means to determine the electron-laser timing using a copper mesh at the target position with an incident fluence of $14.9 \mathrm{~mJ} / \mathrm{cm}^{2}$. Images of the mesh are acquired on the detector in transmission mode. The magnification is close to one, so that the lines with a spacing of $24.5 \mu \mathrm{m}$ are not resolved in these images. Similar experiments have been presented in ${ }^{57}$. Examples of images prior to and after laser irradiation are shown in fig. 8 (a) and (b). The excitation laser with a width of $500 \mu \mathrm{m}$, a wavelength of $800 \mathrm{~nm}$ and an pulse width of $\approx 80$ fs induces

15 a plasma in the center of the mesh, from where a charged plume is expelled. The probe electron beam is partially deflected by this spatial gradient in charge, which can be observed as the depleted area in the electron density distribution, as depicted in fig. 8 (b). The electron beam seems to be affected on a smaller area than expected from the laser beam size, which we interpret as higher order order multiphoton absorption processes lead in turn to reduction in the effective beam diameter giving rise to the observed effect. The excitation arrival time was varied using a delay stage and a series of shadow images was recorded scanning through the time delay between the laser excitation and electron probe pulses. In order to retrieve $t=0$ from the shadow images, we analyzed the RMS intensity changes in the central area of the beam. As plotted in fig. 8(c), we observed a step in the signal, the onset of which is assumed to indicate the zero - time point. Furthermore, we evaluated the width of the differential change in intensity and find a FWHM of 0.9 ps where the changes are occurring fastest in the center of

so the imaged profile. The result indicates that the electron bunch length is in the sub picosecond range.

18 | Faraday Discuss., [year], [vol], 1-25

This journal is @ The Royal Society of Chemistry [year] 

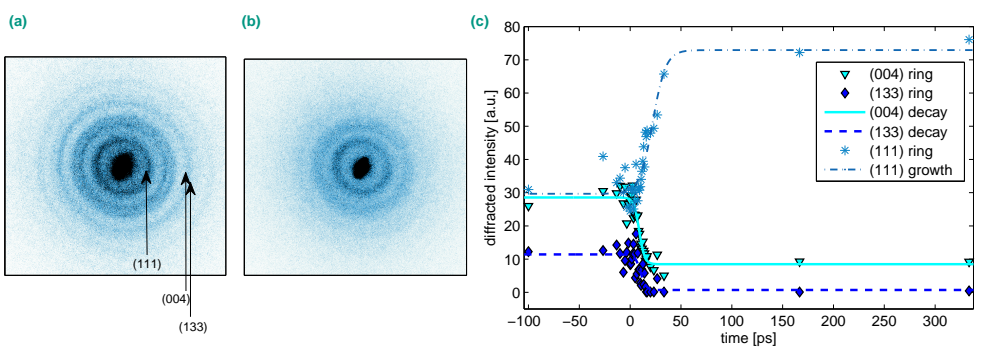

Fig. 9 Single shot diffraction patterns before (a) and after (b) laser excitation. (c) Intensity of the diffraction rings as a function of time delay between pump and probe pulse.

\subsection{Laser induced structural dynamics of Gold}

The first structural dynamics at REGAE were observed in a similar geometry to that used for the plasma studies, but under single shot conditions. Free standing membranes of polycrystalline gold were used to observe a laser induced struc-

5 tural change. The membranes with a size of $200 \mu \mathrm{m}$ by $200 \mu \mathrm{m}$ were excited one by one with a laser pulse, and the resultant diffraction images were recorded. At a fluence of $150 \mathrm{~mJ} / \mathrm{cm}^{2}$ and $100 \mathrm{fs}$ pulse duration, the excitation leads to melting and therefore damage of the sample, and is not reversible. For the data presented here, 300 of these membranes fabricated on a chip were used. Again, example 10 images before and after laser irradiation are shown in fig. 9 (a) and (b). The diffraction image after laser irradiation still exhibits diffraction rings, but the amplitude of the outer rings is diminished. This change indicates a loss of long range order due to melting of the sample. The timescale of this change from ordered to a disordered state is shown in fig. 9(c). Here, the diffraction rings' amplitudes are

15 plotted versus delay time between laser and electron bunch. Each time step was repeated three times. Within five picoseconds after excitation, the outer rings are fading, while the ring indexed with (111) gains in intensity. This gain is attributed to an overlap of the (111) diffraction ring and diffraction from a liquid state. At longer delay time we find the sample completely destroyed. The above results

20 are the first time-resolved studies of REGAE that clearly show its capabilities for single shot structure determination on the $100 \mathrm{fs}$ time scale. The observed dynamics for the particular fluences match very well those found earlier ${ }^{58}$. The present time resolution is limited by RF timing issues and it must be stated that this limit is primarily due to problems in finding a suitably fast reference system to optimize the phase of the rebunching cavity for optimal compression. The best solution will be to introduce an RF streak camera for enabling optimization of the pulse compression process (in progress). This diagnostic tracks the phase jitter of the RF so it is not suitable for jitter compensation between the RF and the laser excitation. However, by simultaneously recording the central beam position with 30 the high dynamic range detector, it will be possible to achieve an overall time resolution in the $10 \mathrm{fs}$ range, as demonstrated using phototriggered streak cameras for time stamping and correction of the jitter ${ }^{26-29}$. 


\section{Concluding Remarks}

The present space-time limitations have been increased to the 10 femtosecond time scale with changes in intensity in diffraction orders corresponding to net atomic displacements of less than $0.05 \AA$ with single shot capabilities. This

5 space-time resolution is sufficient now to capture the fastest nuclear motions involved in chemistry. In this respect, the single most restrictive condition on space-time resolution, not discussed within the context of the electron source physics, is the sample constraints. The use of electron structural probes to provide table top high brightness sources by its nature requires very thin samples,

10 on the order of few $10 \mathrm{~s}$ of nanometers to $100 \mathrm{~nm}$ scale for nonrelativistic electrons. It is the sample limitations that impose the brightness condition in the first place. These are precious samples in which excitation induced damage and thermal effects limiting sampling rate have dictated the need for ultrabright sources. There are some samples however that are just not amenable to such short path-

15 lengths. Two prime examples of great interest are solution phase chemistry and protein dynamics. Although, there have been major advances in the development of nanofluidics for this expressed purpose ${ }^{59,60}$, it is much simpler to use micron scale flow conditions to achieve the desired flow rates for sample exchange. Similarly for protein systems, the highest spatial resolution is achieved 20 in diffraction and the functionally relevant motions involving the protein are spatially distributed leading to very small net RMS atomic motions, yet are central to the proteins role in transducing stored chemical potential into function ${ }^{4}$. If we are ever to make the key connections between correlated motions inherent to particular protein structures or motifs as part of a generalized understanding of

25 protein structure-function correlations, we will need to make these observations in diffraction. All protein crystals are water soluble and as such are not amenable to classic microtome methods to fabricate $100 \mathrm{~nm}$ thin slices needed for nonrelativistic electron probes. Herein is the major advantage of relativistic electrons over lower energy sources. There are scaling relations for the electron mean free 30 path that indicate higher energy electrons will enable the use of thicker samples. However, the difference in elastic and inelastic scattering need to be determined as there are approximations as is evident from the noted differences between experimental measurements and calculated scattering cross-sections. Most important, sample quality has an enormous influence on spatial resolution (vide infra).

35 In the present work, we have conducted a systematic study of the effect of sample thickness on the apparent structure resolution. By comparing both experiment and theoretical calculations based on multi slice methods for dealing with multiple electron scattering, we find that it is possible to use samples up to thicknesses of nearly 1 micron for materials of low $\mathrm{Z}$ corresponding to the range of most 40 organic and biological systems of interest. This finding also means that it should be possible to dramatically relax the engineering requirements for introducing flowing liquid cells with electron transparent $\mathrm{SiN}$ windows. In terms of spatial resolution limits for discerning structural changes, it is important to emphasize further that the measurement does not have the same requirements as needed to 45 resolve a previously unknown structure. The experiment relies on knowing the initial and in most cases final structure and observing the differential displacements in atomic positions that connect one structure to another. This observation 
gives us directly information on the key modes coupled to the reaction coordinate and enables observation of the reduction in dimensionality that occurs in curve cross regions between reactant and product surfaces. This feature is central to chemistry and it is this enormous reduction in dimensionality in barrier or curve 5 crossing regions that ultimately makes chemistry a transferable concept. These relatively small but highly correlated motions are observable as long as the relative ratios of the various diffraction orders is conserved and within the dynamic range of the structurally induced changes in intensity. We therefore conclude that it will be possible to pull out the structural changes for many classes of time10 resolved diffraction experiments, involving samples that were previously thought intractable due to either their liquid nature or sample thickness issues. This finding that multiple electron scattering is not as big an effect as sample quality and attenuation with sample thickness has enormous implications for improving sample preparation methods. This study also reaffirms the primary motivation for the 15 development of relativistic electron sources for atomically resolved structural dynamics as this source greatly relaxes sample requirements for all classes of study. Future investigations comparing data to calculated diffraction patters will give more insight into the sensitivity that is required to further confirm our observation.

20 In terms of space-time resolution, relativistic sources also offer the highest possible source brightness for a given photocathode. The high-Q RF cavities provide the highest possible field gradients to accelerate the electron bunch up to the relativistic regime prior to any excessive space-charge broadening effects can spoil the space time correlation of the electrons in the accelerating field. This 25 condition is central to the use of rebunching cavities to correct the small velocity spread from the field gradient. As shown in the present work, based largely on well tested simulations, pulses as short as $10 \mathrm{fs}$ with electron bunch charges of $10^{6}-10^{7}$ electrons per pulse for typical matching electron beam to laser excitation beam parameters are achievable. It should be noted that there is a great deal of so effort to further increase field gradients for next generation electron accelerators and light sources. The use of extremely high peak power few cycle $\mathrm{THz}$ pulses has recently been demonstrated to give uniform acceleration and deceleration of nonrelativistic seed electrons with estimated field gradients in excess of $1 \mathrm{GV} / \mathrm{m}$ or more than an order of magnitude larger than the best RF cavities ${ }^{61}$. This 35 development would lead to correspondingly shorter electron pulses for a given bunch density. With respect to improving time resolution, concepts similar to streaking with RF deflecting structures might be implemented in the future as well ${ }^{62}$.

The most important advances in electron source brightness will deal with the transverse coherence limitations. The present photocathodes typically have initial transverse energy spreads of 0.2 to $0.6 \mathrm{eV}$. This initial energy spread is the greatest limiting factor in the transverse coherence and ability to image complex molecular systems such as proteins. Even within this range of transverse momentum spread, it has been possible to now track atomic motions for large molecular 45 systems with unit cells of $>3 \mathrm{~nm}$ that are comparable to unit cells of many important protein systems ${ }^{63}$. Further decreases in transverse momentum spread will make higher resolution of even minute motions as well as scaling to larger and more complex systems. In this respect, the use of ultracold atom sources using 
threshold ionization of Rydberg states have given results close to the theoretical quantum limit for fermions with respect to the minimum transverse velocity spread, yielding effective electron temperatures of $10 \mathrm{~K}$ or less than $0.6 \mathrm{meV}^{41}$. This approach, however, is limited in electron density by the relatively low atom 5 density achievable in ultracold atom traps. In order to get sufficient electron numbers for near single shot conditions, the initial beam size approaches $\mathrm{mm}$ dimensions that cancel the net reduction in emittance. The electron beam is indeed very cold but the angular distribution does not lead to much improvement in the transverse coherence at the sample position due to the very large source

10 size at the photocathode. We contend that the brightest photocathodes will still be based on solid state cathodes. Significant progress has been made lately in understanding the scattering issues and boundary conditions for photoemission from solid state systems. Recent studies of trialkali photocathodes have given evidence for thermally limited photoemission with energy spreads of $160 \mathrm{meV}$ at 15 room temperature ${ }^{64}$, and factors of 10 reduction are clearly possible with excitation closer to threshold. These photocathodes also enjoy relative high quantum yields as found for other semiconductor photocathodes such as the routinely used CsTe materials. Although it is not straightforward, it should be possible to use cryogenically cooled solid state photocathodes. The challenge is to thermally 20 isolate the cathode section of the electron gun while still having sufficient conduction to avoid excessive charge build up and break down at the high fields used for extraction. It should nevertheless be possible to go to $10 \mathrm{~K}$ for suitable source sizes that would give an increase in source brightness of approximately 2 orders of magnitude over the commonly used photocathodes in femtosecond electron 25 diffraction studies. In this event, it will be possible to scale up the experiments to the study of effectively any molecular system and relevant biological systems.

The above technical achievements could be made in the relatively near term. As with all experiments aimed at structure determination, the quality of the sample ultimately dictates the limits. Future efforts will need to focus on new meth30 ods of introducing samples into the vacuum environment of electron diffraction or real space imaging systems. With the relatively recent advent of nanotechnology into this domain, there is reason to believe that it will soon be possible to introduce large arrays of crystals, with the potential for M-pixel arrays of nanocrystals into the sample chambers. High throughput methods developed for

35 XFEL experiments could also be adopted for electron use with appropriate design of environmental enclosures around the sample viewing area. Ion milling and in situ growth of 2D crystals and nanocrystals will need to be explored ${ }^{19}$. In all cases, the lessons learned in increasing source brightness will pay enormous dividends for increasing the spatial resolution using electrons for static structure 40 determinations and the importance of these advances should not be undervalued. In the end, it will be the sample that will dictate the space-time resolution limits - as it should be.

\section{Acknowledgements}

We gratefully acknowledge the support received for this collaborative effort by 45 DESY (member of the Helmholtz Association (HGF)), the Max Planck Society, and the Hamburg Centre for Ultrafast Imaging, CUI (DFG-EXC1074). We are in- 
debted to R. Brinkmann and H. Dosch of DESY for supporting this project. Also, we thank Hamburg University (UHH) for supporting the setup of the experiment as well as the MCS, MIN, MKK, MSK, MVS groups of DESY for countless support during setup and experiment. In this respect we particularly like to thank 5 J. Müller, K. Müller, M. Huening, J. Herrmann, R. Jonas, U. Mavric R. Kammering, T. Kozak, A. Labudda, U. Laustoer, S. Lederer, L. Lilje, D. Lipka, I. Peperkorn, S. Pfeiffer, H. Schlarb, C. Schmidt, M. Seebach, M. Titberidze, M. Walla, S. Weisse, and K. Witt for great support. T. Hasegawa thanks J. K. Dewhurst for providing EDiCo electron diffraction code and his technical support.

10 We are also grateful for technical support from D. Gitaric and J. Gonschior.

\section{References}

1 J. C. Polanyi and A. H. Zewail, Accounts of Chemical Research, 1995, 28, 119-132.

2 J. R. Dwyer, C. T. Hebeisen, R. Ernstorfer, M. Harb, V. B. Deyirmenjian, R. E. Jordan and R. J. D. Miller, Philosophical transactions. Series A, Mathematical, physical, and engineering sciences, 2006, 364, 741-78.

3 B. J. Siwick, J. R. Dwyer, R. E. Jordan and R. J. D. Miller, Science (New York, N.Y.), 2003, 302, 1382-5.

4 R. J. D. Miller, Annual review of physical chemistry, 2014, 65, 583-604.

5 M. Gao, C. Lu, H. Jean-Ruel, L. C. Liu, A. Marx, K. Onda, S.-y. Koshihara, Y. Nakano, X. Shao, T. Hiramatsu, G. Saito, H. Yamochi, R. R. Cooney, G. Moriena, G. Sciaini and R. J. D. Miller, Nature, 2013, 496, 343-6.

6 M. J. J. Vrakking and T. Elsaesser, Nature Photonics, 2012, 6, 645-647.

7 P. Emma, R. Akre, J. Arthur, R. Bionta, C. Bostedt, J. Bozek, A. Brachmann, P. Bucksbaum, R. Coffee, F.-J. Decker, Y. Ding, D. Dowell, S. Edstrom, A. Fisher, J. Frisch, S. Gilevich, J. Hastings, G. Hays, P. Hering, Z. Huang, R. Iverson, H. Loos, M. Messerschmidt, A. Miahnahri, S. Moeller, H.-D. Nuhn, G. Pile, D. Ratner, J. Rzepiela, D. Schultz, T. Smith, P. Stefan, H. Tompkins, J. Turner, J. Welch, W. White, J. Wu, G. Yocky and J. Galayda, Nature Photonics, 2010, 4, 641-647.

8 A. Barty, J. Küpper and H. N. Chapman, Annual review of physical chemistry, 2013, 64, 415-35.

309 P. Hänggi and M. Borkovec, Reviews of Modern Physics, 1990, 62, 251-341.

10 D. D. Dlott, J. Opt. Soc. Am. B, 1990, 7, 1638-1652.

11 R. J. D. Miller, Annual review of physical chemistry, 1991, 42, 581-614.

12 P. Altoè, A. Cembran, M. Olivucci and M. Garavelli, PNAS, 2010, 107, 20172-20177.

13 V. I. Prokhorenko, A. Halpin, P. J. M. Johnson, R. J. D. Miller and L. S. Brown, The Journal of chemical physics, 2011, 134, 085105.

14 R. J. D. Miller, Science (New York, N.Y.), 2014, 343, 1108-16.

15 M. Harmand, R. Coffee, M. R. Bionta, M. Chollet, D. French, D. Zhu, D. M. Fritz, H. T. Lemke, N. Medvedev, B. Ziaja, S. Toleikis and M. Cammarata, Nature Photonics, 2013, 7, 215-218.

16 A. Aquila, M. S. Hunter, R. B. Doak, R. a. Kirian, P. Fromme, T. a. White, J. Andreasson, D. Arnlund, S. Bajt, T. R. M. Barends, M. Barthelmess, M. J. Bogan, C. Bostedt, H. Bottin, J. D. Bozek, C. Caleman, N. Coppola, J. Davidsson, D. P. DePonte, V. Elser, S. W. Epp, B. Erk, H. Fleckenstein, L. Foucar, M. Frank, R. Fromme, H. Graafsma, I. Grotjohann, L. Gumprecht, J. Hajdu, C. Y. Hampton, A. Hartmann, R. Hartmann, S. Hau-Riege, G. Hauser, H. Hirsemann, P. Holl, J. M. Holton, A. Hömke, L. Johansson, N. Kimmel, S. Kassemeyer, F. Krasniqi, K.-U. Kühnel, M. Liang, L. Lomb, E. Malmerberg, S. Marchesini, A. V. Martin, F. R. N. C. Maia, M. Messerschmidt, K. Nass, C. Reich, R. Neutze, D. Rolles, B. Rudek, A. Rudenko, I. Schlichting, C. Schmidt, K. E. Schmidt, J. Schulz, M. M. Seibert, R. L. Shoeman, R. Sierra, H. Soltau, D. Starodub, F. Stellato, S. Stern, L. Strüder, N. Timneanu, J. Ullrich, X. Wang, G. J. Williams, G. Weidenspointner, U. Weierstall, C. Wunderer, A. Barty, J. C. H. Spence and H. N. Chapman, Optics express, 2012, 20, 2706-16.

17 E. Möhr-Vorobeva, S. L. Johnson, P. Beaud, U. Staub, R. De Souza, C. Milne, G. Ingold, J. Demsar, H. Schaefer and A. Titov, Physical Review Letters, 2011, 107, 036403.

18 A. Zarrine-Afsar, C. Müller, F. O. Talbot and R. J. D. Miller, Analytical chemistry, 2011, 83, $767-73$. 
19 A. Zarrine-Afsar, T. R. M. Barends, C. Müller, M. R. Fuchs, L. Lomb, I. Schlichting and R. J. D. Miller, Acta crystallographica. Section D, Biological crystallography, 2012, 68, 321-3.

20 J. C. H. Spence, U. Weierstall and H. N. Chapman, Reports on progress in physics. Physical Society (Great Britain), 2012, 75, 102601.

521 A. H. Zewail, Annual review of physical chemistry, 2006, 57, 65-103.

22 P. Baum and A. H. Zewail, Proceedings of the National Academy of Sciences of the United States of America, 2006, 103, 16105-10.

23 B. J. Siwick, J. R. Dwyer, R. E. Jordan and R. J. D. Miller, Journal of Applied Physics, 2002, 92, 1643.

1024 T. van Oudheusden, E. F. de Jong, S. B. van der Geer, W. P. E. M. O. t Root, O. J. Luiten and B. J. Siwick, Journal of Applied Physics, 2007, 102, 093501.

25 L. Veisz, G. Kurkin, K. Chernov, V. Tarnetsky, a. Apolonski, F. Krausz and E. Fill, New Journal of Physics, 2007, 9, 451-451.

26 M. Gao, H. Jean-Ruel, R. R. Cooney, J. Stampe, M. D. Jong, M. Harb, G. Sciaini, G. Moriena and R. J. D. Miller, Opt. Express, 2012, 20, 799-802.

27 R. P. Chatelain, V. R. Morrison, C. Godbout and B. J. Siwick, Applied Physics Letters, 2012, 101, 081901.

28 R. P. Chatelain, V. Morrison, C. Godbout, B. van der Geer, M. de Loos and B. J. Siwick, Ultramicroscopy, 2012, 116, 86-94.

2029 M. Gao, Y. Jiang, G. H. Kassier and R. J. Dwayne Miller, Applied Physics Letters, 2013, 103, 033503.

30 T. Elsaesser and M. Woerner, Acta crystallographica. Section A, Foundations of crystallography, 2010, 66, 168-78.

31 V. Juvé, M. Holtz, F. Zamponi, M. Woerner, T. Elsaesser and a. Borgschulte, Physical Review Letters, 2013, 111, 217401.

32 M. Woerner, F. Zamponi, Z. Ansari, J. Dreyer, B. Freyer, M. Prémont-Schwarz and T. Elsaesser, The Journal of chemical physics, 2010, 133, 064509.

33 J. B. Hastings, F. M. Rudakov, D. H. Dowell, J. F. Schmerge, J. D. Cardoza, J. M. Castro, S. M. Gierman, H. Loos and P. M. Weber, Applied Physics Letters, 2006, 89, 184109.

3034 P. Musumeci, J. T. Moody, C. M. Scoby, M. S. Gutierrez and M. Westfall, Applied Physics Letters, 2010, 97, 063502.

35 R. Li, W. Huang, Y. Du, L. Yan, Q. Du, J. Shi, J. Hua, H. Chen, T. Du, H. Xu and C. Tang, The Review of scientific instruments, 2010, 81, 036110.

36 P. Musumeci, J. T. Moody, C. M. Scoby, M. S. Gutierrez, M. Westfall and R. K. Li, Journal of Applied Physics, 2010, 108, 114513.

37 C. M. Scoby, P. Musumeci, J. T. Moody and M. S. Gutierrez, Physical Review Special Topics Accelerators and Beams, 2010, 13, 022801.

38 Y. Murooka, N. Naruse, S. Sakakihara, M. Ishimaru, J. Yang and K. Tanimura, Applied Physics Letters, 2011, 98, 251903.

4039 A. M. Michalik, E. Y. Sherman and J. E. Sipe, Journal of Applied Physics, 2008, 104, 054905.

40 G. Sciaini and R. J. D. Miller, Reports on Progress in Physics, 2011, 74, 096101.

41 W. J. Engelen, M. a. van der Heijden, D. J. Bakker, E. J. D. Vredenbregt and O. J. Luiten, Nature communications, 2013, 4, 1693.

42 A. Rother and K. Scheerschmidt, Ultramicroscopy, 2009, 109, 154-60.

4543 L. Reimer and H. Kohl, Transmission Electron Microscopy, Springer, 2008.

44 K. Dewenhurst and D. Rankin, EDICo - Electron diffraction code, http://edico.sourceforge.net/.

45 R. F. Egerton, Phys. Status. Solidi (a), 1976, 663, 663-668.

46 B. Kabius, P. Hartel, M. Haider, H. Müller, S. Uhlemann, U. Loebau, J. Zach and H. Rose, Journal of electron microscopy, 2009, 58, 147-55.

5047 CST STUDIO SUITE, CST Computer Simulation Technology AG, www.cst.com.

48 M. Reiser, Theory and Design of Charged Particle Beams, Wiley, 2nd edn, 2008.

49 R. K. Li and P. Musumeci, Physical Review Applied, 2014, 2, 024003.

50 D. Xiang, F. Fu, J. Zhang, X. Huang, L. Wang, X. Wang and W. Wan, Nuclear Instruments and Methods in Physics Research Section A: Accelerators, Spectrometers, Detectors and Associated Equipment, 2014, 759, 74-82.

51 H. Delsim-Hashemi and K. Flöttmann, Proceedings of IPAC2014, Dresden, Germany, 2014.

52 D. Lipka and M. Seebach, Proceedings of IBIC2013, 2013, 872-875. 
53 S. Bayesteh and K. Floettmann, Proceedings of IPAC2014, Dresden, Germany, 2014, pp. 35153517.

54 M. Hoffmann, H. Kay, U. Mavric, H. Schlarb, C. Schmidt, W. Jalmuzna, T. Kozak and A. Piotrowski, Proceedings of IPAC2013, Shanghai, China, 2013, pp. 2938-2940.

555 K. Floettmann, ASTRA - A Space Charge Tracking Algorithm, http://www.desy.de/ mpyflo/, 1997-2014.

56 CrystalMaker Software Ltd, www.crystalmaker.com.

57 C. M. Scoby, R. K. Li and P. Musumeci, Ultramicroscopy, 2013, 127, 14-8.

58 R. Ernstorfer, M. Harb, C. T. Hebeisen, G. Sciaini, T. Dartigalongue and R. J. D. Miller, Science (New York, N.Y.), 2009, 323, 1033-7.

59 J. M. Grogan, N. M. Schneider, F. M. Ross and H. H. Bau, Journal of the Indian Institute of Science, 2012, 92, 295.

60 C. Mueller, M. Harb, J. R. Dwyer and R. J. D. Miller, The Journal of Physical Chemistry Letters, 2013, 4, 2339-2347.

1561 S. Carbajo, W. L. J. Nanni, E. A., R. J. D. Miller and F. X. Kärtner, submitted, 2014.

62 K. Floettmann and V. V. Paramonov, Phys. Rev. ST Accel. Beams, 2014, 17, 024001.

63 S. Hayes, in preparation, 2014.

64 L. Cultrera, I. Bazarov, A. Bartnik, B. Dunham, S. Karkare, R. Merluzzi and M. Nichols, Applied Physics Letters, 2011, 99, 152110. 